\title{
Is It Real False Negative Finding in Motor Evoked Potential Monitoring during Corrective Surgery of Ankylosing Spondylitis? A Case Report
}

\author{
Ki-Tack Kim¹, Sang-Hun Lee ${ }^{1}$, Yoon-Ho Kwack², Eon-Seok Son ${ }^{3}$ \\ 'Department of Orthopedic Surgery, Kangdong Hospital, Kyung Hee University School of Medicine, Seoul, Korea \\ ${ }^{2}$ Department of Orthopedic Surgery, Bumin Hospital, Seoul, Korea \\ ${ }^{3}$ Department of Medicine, Graduate School, Kyung Hee University School of Medicine, Seoul, Korea
}

\begin{abstract}
We performed L1 posterior vertebral columnar resection and posterior correction for Andersson's lesion and thoracolumbar kyphosis in an ankylosing spondylitis patient during motor evoked potential (MEP) monitoring. We checked MEP intra-operatively, whenever a dangerous procedure for neural elements was performed, and no abnormal findings were seen during surgery. After the operation, we examined neurologic function in the recovery room; the patient showed a progressive neurologic deficit and no response to MEP. After emergency neural exploration and decompression surgery, the neurologic deficit was recovered. We questioned whether to acknowledge the results of this case as a false negative. We think the possible reason for this result may be delayed development of paralysis. So, we recommend that MEP monitoring should be performed not only after important operative steps but also after all steps, including skin suturing, for final confirmation.
\end{abstract}

Key Words: Spine operation, Deformity correction, Motor evoked potential, Delayed paraplegia

\section{Introduction}

As modalities that monitor neurologic deficits during operation of spinal deformity, somatosensory evoked potentials (SSEPs) and motor evoked potentials (MEPs) are currently available. Among others, SSEPs have been reported with false negative results by several authors $[1,2]$. Theoretically, however, negative findings cannot be observed with MEPs after severe injury to the central nervous system. In fact, reports on cases that demonstrated paralysis after normal MEP findings during the operation of spinal deformity are very rare, and the etiology remains unclear. Against this background, we report a clinical case in which a patient developed paraplegia after normal MEP findings and review it with previously reported cases.

\section{Case Report}

A 58-year-old male patient, diagnosed with ankylosing spondylitis 20 years ago, complained of persistent pain and kyphosis in the thoracolumbar area. Non-union between $\mathrm{T} 12$ and L1 and a $50^{\circ}$ thoracolumbar kyphosis centered on this area were observed with radiologic evaluations (Fig. 1). No neurologic deficits were found in physical examinations.

Under intravenous anesthesia using Fresopol and Remifentanil, the patient was placed in a prone position, and electrodes were connected for MEP and electromyography (EMG) monitoring. The operation was performed via posterior-only approach, and pedicle screws were inserted from T10 to L4 except for L1. Posterior vertebral columnar resection (PVCR) was performed on L1, and an auto bone was transplanted in the MESH cage, which was placed in

Received May 2, 2011; Revised Jun 20, 2011; Accepted Jun 23, 2011

Corresponding author: Yoon-Ho Kwack, MD

Department of Orthopedic Surgery, Bumin Hospital,

657 Deungchon 3-dong, Gangseo-gu, Seoul 157-930, Korea

Tel: +82-2-2620-0003, Fax: +82-2-2620-0130, E-mail: kh21635@hanmail.net 
the vertebral column between T12 and L2. Then, correction to reduce the kyphotic angle from the posterior aspect was done. A device that monitors neurologic deficits, NIM-SPINETM (Medtronic, Sofamor Danek, Memphis, TN, USA), which allowed both transcranial electrical stimulation (motor evoked potentials) and continuous EMG monitoring, was used. Two electrodes were inserted into the scalp to provide transepidermal electric stimulation. Recording electrodes were placed in the motor nerves of the left and right upper and lower limbs to confirm responses to the stimulation. Intraoperatively, EMG was continuously monitored. MEP monitoring was made at the operative steps that put neural structures at risk for injury: before skin incision, after insertion of pedicle screws, during PVCR, and before and after correction of the kyphosis. The last MEP monitoring was performed after final tightening of pedicle screws. Throughout the procedures, MEP findings were normal. There was an intraoperative estimated blood loss of $800 \mathrm{ml}$, and his blood pressure fell to 62/52 $\mathrm{mm} \mathrm{Hg}$ at the end of surgery from $110 / 60 \mathrm{~mm} \mathrm{Hg}$. His body temperature ranged between $36.6-37.2^{\circ} \mathrm{C}$ as well. Afterwards, safe operative techniques, such as irrigation of the operation area, bone grafting, and suture of the incised area were applied. The patient was subsequently awakened from anesthesia and transferred to the recovery room (RR).

The neurologic examination made in the RR presented a significant decrease at grade I for right big toe and ankle dorsiflexion. The left side also demonstrated a decrease to grade III, followed by reduced sensation. Such neurologic deficits were progressive, and an hour later, sensory and motor functions of both lower limbs were all lost. Therefore, MEP monitoring was applied again, but no waves at all were observed. In addition, 3-dimensional computed tomography did not reveal any misplacement of pedicle screws or bone fragments that might have caused paralysis.

As the patient did not recover from paraplegia even after two hours, he was sent back to the operation room for neurologic exploration. Nerve compression or injury was not observed. Thus, posterior decompression was performed on T12, an area with the highest risk of compression, and the correction angle was reduced. Then, an additional pedicle screw was inserted into L5 and fixed (Fig. 2). As with the primary operation, the patient was put under intravenous anesthesia and monitored for MEP and EMG. With MEP examination after removing the rod to reduce the correction angle, there was a sign of wave to the right (Fig. 3). The patient subsequently presented an evident wave of MEP continuously, and the operation was concluded. From 12 hours after the second operation, motor and sensory functions of the lower limb recovered. At postoperative week 1 , sensory function was fully recovered, and at week 3 , ambulation was possible with the help of a walker. At 3 months after primary and secondary operations, the muscle strength and sense of the lower limb were normal, and independent ambulation was possible.

\section{Discussion}

The modalities applied to monitor neurologic deficits during spinal surgery include the wake up test, SSEPs,
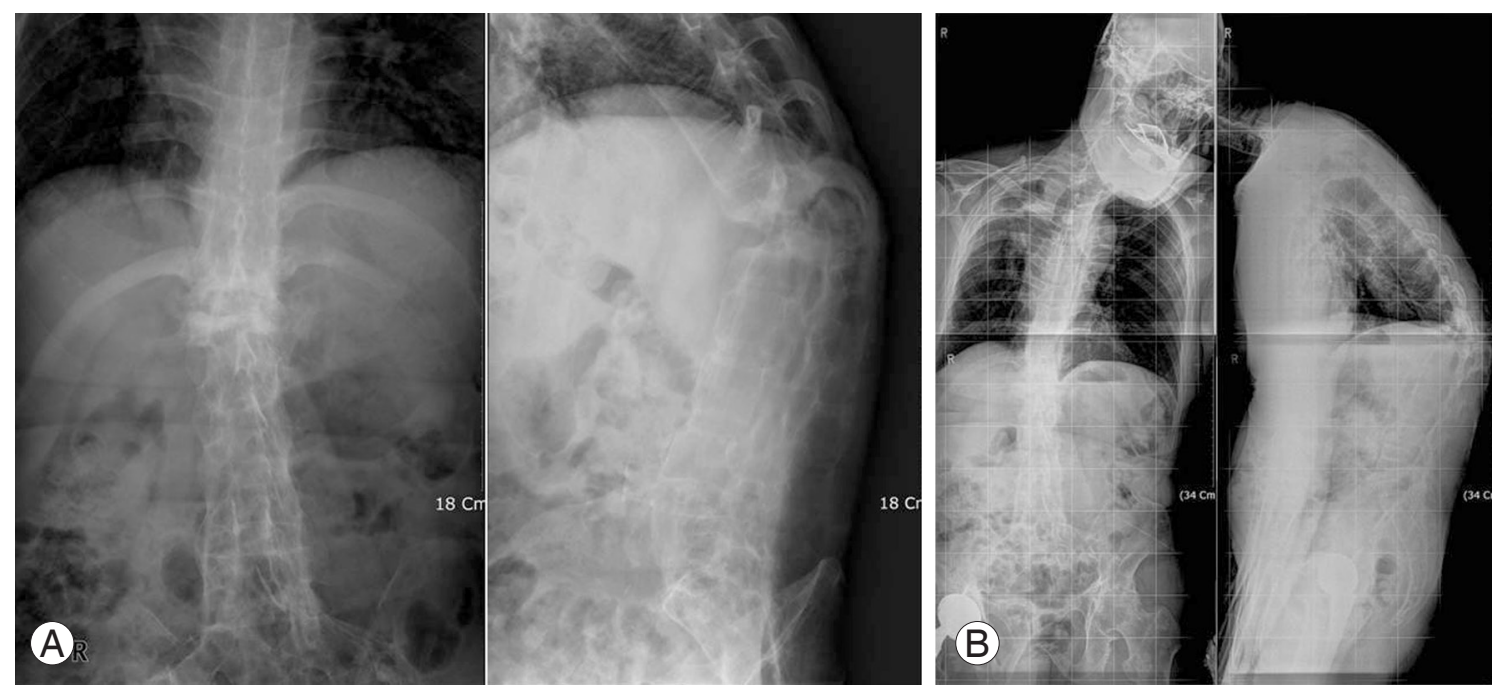

Fig. 1. Preoperative radiogram (58-year-old male, ankylosing spondylitis with kyphosis and Andersson's lesion). (A) Anteroposterior and lateral radiogram, which exhibited Andersson's lesion between T12 and L1. (B) Whole spine radiogram, which exhibited kyphotic angle of $50^{\circ}$ and left side bending in the cervicothoracic region. 
and MEPs. The wake up test had been the only method to detect injury of the corticospinal tract and motor functions intraoperatively before MEPs were introduced. It has the advantage that checking conditions of the patient's physiologic motor functions is possible. However, the test is not in use currently, due to its disadvantages, such as a delay in operative time, discontinuous assessment of neural functions that does not provide an operative step during which injury occurred, and limited application to patients with cognitive or hearing deficits.

SSEPs have been widely accepted since the early 1970s as a tool to identify the presence of neurologic injury by monitoring the dorsal column-medial lemniscus pathway. However, SSEPs do not provide monitoring of the spinothalamic pathway involved with pain and temperature and the corticospinal tract related to motor functions. Furthermore, direct injury to the anterior and lateral spinal cord or reduced blood flow cannot be observed with SSEPs
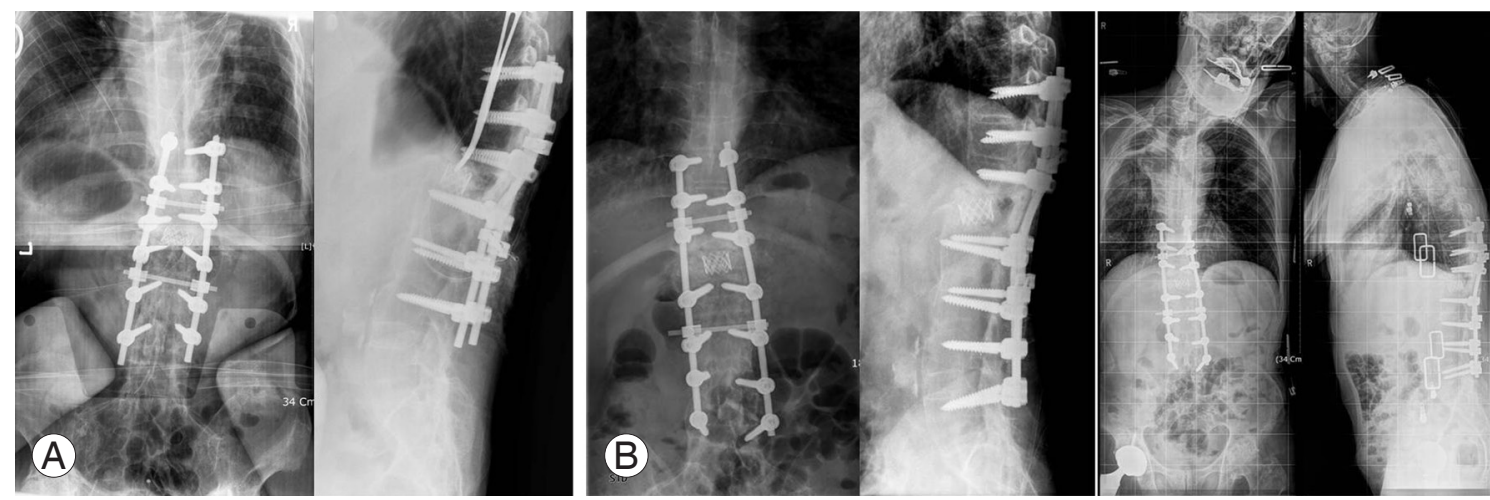

Fig. 2. (A) Primary postoperative anteroposterior radiogram, which exhibits a decreased kyphotic angle. But, we planned an emergency reoperation because the patient showed progressive paraplegia. (B) Anteroposterior radiogram after secondary surgery. After operating, walking was possible after 3 weeks.
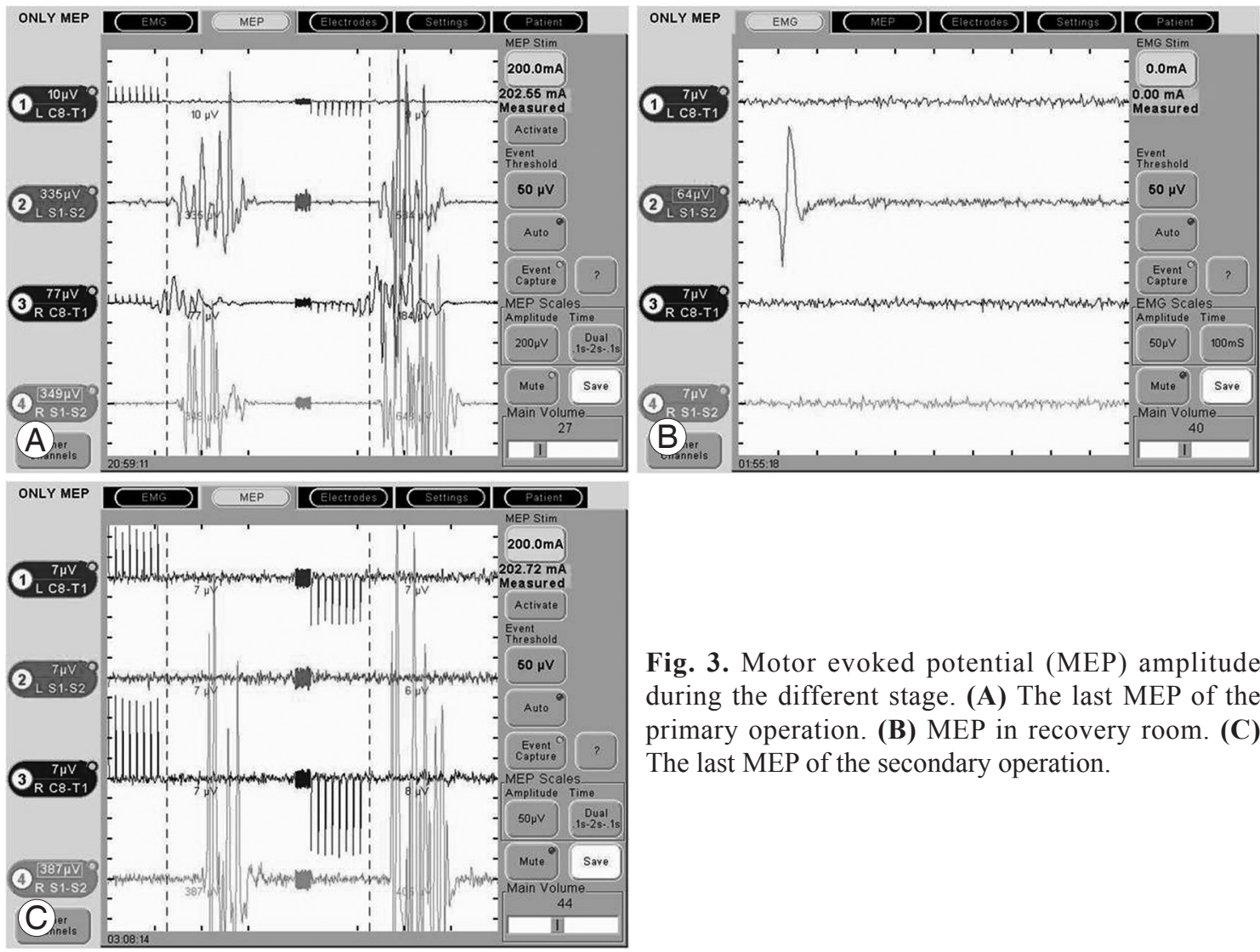

Fig. 3. Motor evoked potential (MEP) amplitude during the different stage. (A) The last MEP of the primary operation. (B) MEP in recovery room. (C) The last MEP of the secondary operation. 
[3]. For these reasons, several authors have reported false negative results in their clinical studies [1,2].

MEPs were first introduced in 1980 by Merton and Morton [4] to overcome the disadvantages of SSEPs in monitoring neurologic deficits intraoperatively. As MEPs can identify changes in the corticospinal tract with a higher sensitivity to reduced motor functions than SSEPs, MEP monitoring has been commonly used in recent years [5-7]. However, it does not provide continuous assessment during operation, encouraging attempts to apply the technique in combination with other modalities, such as $\mathrm{D}$ wave or EMG, in an effort to overcome the limitation and increase sensitivity. Among others, D wave was found highly relevant to clinical results, but it cannot be applied to the thoracolumbar junction below T11 or to the lumbar area [8]. The combined use of MEPs and EMG was also reported by several authors with good results. Skinner et al. [9] even described a sensitivity of up to $100 \%$. However, false negative results of MEPs have rarely been reported. In a recent operation performed with MEPs and EMG, Modi et al. [10] reported false negative clinical results but failed to describe the change of MEPs according to the time elapsed and neurologic changes of the patient. Without clarifying the reason, they suggested significant blood loss during the operation and hypotension as probable reasons for the results [10].

We first questioned whether to acknowledge the results of this case as false negative. In other words, it was theoretically impossible to demonstrate normal findings on both MEPs and EMG when a problem in nerve conduction has occurred. Therefore, we found it unacceptable to consider the results as false negative. The possible reasons for the results in our case may be ischemia, compression, or distraction of the spinal cord, any of these may have caused changes in neural symptoms and MEPs when slight compression or a blood flow disorder was provided to the spinal cord persistently rather than rapidly. Whatever the delayed symptoms and changes in MEPs after skin suture and recovery from anesthesia because of reason was, the case in our study manifested some problems that developed gradually with time, although his findings on the final MEPs were normal after posterior correction.

To date, no studies have focused on MEP results that change over time after persistent neural stimulation and injury. Thus, we cannot conclude that the delayed development of paralysis in this case was the result of minor but persistent stimulation that could not be detected by MEPs. In this regard, studies on effects of minor stimulation or blood flow disorder on MEP results with time are considered necessary. In addition, we recommend that MEP monitoring be performed not only after important operative steps but also after all steps, including skin suturing, for final confirmation and prevention of the results as described in this case.

\section{REFERENCES}

1. Ben-David B, Haller G, Taylor P. Anterior spinal fusion complicated by paraplegia: a case report of a falsenegative somatosensory-evoked potential. Spine (Phila Pa 1976) 1987;12:536-9.

2. Jones SJ, Buonamassa S, Crockard HA. Two cases of quadriparesis following anterior cervical discectomy, with normal perioperative somatosensory evoked potentials. J Neurol Neurosurg Psychiatry 2003;74:2736.

3. Gonzalez AA, Jeyanandarajan D, Hansen C, Zada G, Hsieh PC. Intraoperative neurophysiological monitoring during spine surgery: a review. Neurosurg Focus 2009;27:E6.

4. Merton PA, Morton HB. Stimulation of the cerebral cortex in the intact human subject. Nature 1980;285:227.

5. Bose B, Sestokas AK, Schwartz DM. Neurophysiological monitoring of spinal cord function during instrumented anterior cervical fusion. Spine J 2004;4:202-7.

6. Hilibrand AS, Schwartz DM, Sethuraman V, Vaccaro AR, Albert TJ. Comparison of transcranial electric motor and somatosensory evoked potential monitoring during cervical spine surgery. J Bone Joint Surg Am 2004;86:1248-53.

7. Hsu B, Cree AK, Lagopoulos J, Cummine JL. Transcranial motor-evoked potentials combined with response recording through compound muscle action potential as the sole modality of spinal cord monitoring in spinal deformity surgery. Spine (Phila Pa 1976) 2008;33:1100-6.

8. Sala F, Palandri G, Basso E, et al. Motor evoked potential monitoring improves outcome after surgery for intramedullary spinal cord tumors: a historical control study. Neurosurgery 2006;58:1129-43.

9. Skinner SA, Nagib M, Bergman TA, Maxwell 
RE, Msangi G. The initial use of free-running electromyography to detect early motor tract injury during resection of intramedullary spinal cord lesions. Neurosurgery 2005;56(2 Suppl):299-314.
10. Modi HN, Suh SW, Yang JH, Yoon JY. False-negative transcranial motor-evoked potentials during scoliosis surgery causing paralysis: a case report with literature review. Spine (Phila Pa 1976) 2009;34:E896-900. 